\title{
AJUSTE RAZONABLE DEL MODELO DE TERAPIA COGNITIVA DE AARON BECK EN UNA MUESTRA PILOTO DE PERSONAS SORDAS SEÑANTES, UNA SISTEMATIZACIÓN DE EXPERIENCIA
}

\section{REASONABLE FIT OF AARON BECK'S COGNITIVE THERAPY MODEL IN A PILOT SAMPLE OF SIGNING DEAF PEOPLE, A SYSTEMATIZATION OF EXPERIENCE}

\author{
Luisa Fernanda Suarez Monsalve \\ Corporación universitaria minuto de Dios UNIMINUTO. Bogotá. Colombia \\ ORCID: https://orcid.org/0000-0003-2415-594X
}

Manuel Alejandro Guerrero Clavijo

Traductor, Universidad de Antioquia, Colombia ORCID: https://orcid.org/0000-0001-8309-0344

\author{
Alis Dahiana Bedoya Olaya \\ Psicóloga, Corporación universitaria minuto de Dios UNIMINUTO. Bogotá. Colombia \\ ORCID: https://orcid.org/0000-0002-4756-5987
}

Cómo referenciar este artículo/How to reference this article:

Suarez Monsalve, L. F., Guerrero Clavijo, M. A. y Bedoya Olaya, A. D. (2021). Ajuste razonable del modelo de terapia cognitiva de Aaron Beck en una muestra piloto de personas sordas señantes, una sistematización de experiencia. Revista de Psicoterapia, 32(118), 217-236. https://doi.org/10.33898/ rdp.v32i118.464

Nota del autor

Aunque la institución a la cual se afilió el estudio no contaba en su momento con un comité ético, se buscó garantizar el principio de anonimato de los participantes voluntarios, aceptando un consentimiento informado digital en LSC.

Desde la ley colombiana 1090 de 2006 se cumplen los artículos 50 y 55. En relación con la resolución 8430 se presenta un riesgo mínimo dando cumplimiento a los artículos 4, 5, 6, 8, 9, 10,12, 14, 26, conforme a los criterios del Artículo 6: Se ajustó a los principios éticos necesarios para el estudio. Para ninguno de los sujetos se contaba con representante legal ya que cada uno de ellos era mayor de edad.

Desde el capítulo tercero de la resolución 8430 de 1993 asociado a las investigaciones en menores de edad o discapacitados, artículo 25, se aclara que no se encontró personal capacitado y con experiencia en Colombia para evaluar sobre la capacidad de entendimiento, razonamiento y lógica del sujeto sordo por lo que no se da cumplimiento a dichos requisitos.

Fecha de recepción v1: 26-11-2020. Fecha de recepción v2: 6-1-2021. Fecha de aceptación: 22-1-2021.

Correspondencia sobre este artículo:

E-mail: psicologacognitivaluisa@gmail.com

(C) 2021 Revista de Psicoterapia

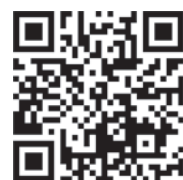




\title{
Resumen
}

La presente sistematización de experiencia planteó como objetivo principal explicar el proceso de ajuste razonable del modelo de terapia cognitiva de Aaron Temkin Beck a una muestra no probabilística de 16 personas sordas, con diferentes niveles comunicacionales, usuarias de lengua de señas colombiana. Partiendo de las características sociolingüísticas para esta población minoritaria, se efectuó un proceso de traducción y retrotraducción de este modelo, con las diferentes distorsiones cognitivas mediante formatos audiovisuales; logrando diseñar una herramienta explicativa, que permitió la comprensión de dicho modelo en población sorda con competencia comunicacional amplia y suficiente.

Palabras Clave: sordera, lengua de señas, psicoterapia, cognición, discapacidad

\begin{abstract}
The main objective of this systematization of experiences was to illustrate the process of adjustment ofAaron Temkin Beck's Cognitive Therapy Model to a non-probability sample of 16 deaf individuals with different levels of communication, users of Colombian Sign Language. Considering the sociolinguistic characteristics of this minority population, a process of translation, backward translation was carried out for this model, with the different cognitive distortions through audiovisual formats. To accomplish this, a tool was designed to allow the understanding of this model in deaf individuals with broad and sufficient communication competence.
\end{abstract}

Keywords: deafness, sign language, therapy, cognition, disability 
Contar con un grado óptimo de salud, ser atendidos oportunamente con calidad y accesibilidad es un derecho fundamental (OMS, 2017), esto incluye la psicoterapia, comprendida como una práctica social y de la salud. Por medio de esta se logra que personas superen crisis emocionales, mejoren relaciones interpersonales, y sea favorecida su salud mental (APA, 2012), como en el caso de pacientes con algún trastorno mental que con ayuda de modelos de intervención como el cognitivoconductual y farmacoterapia, logran reestablecerse o experimentar mejoría (Bogiaizian, 1998), también para el tratamiento de trastornos mentales se encuentra la terapia cognitiva propuesta por Aaron T. Beck, que ha tenido múltiples traducciones incluso a lengua castellana (Beck et al., 1979). Aun así, a la hora de realizar una traducción-adaptación de su modelo junto con las distorsiones cognitivas a la lengua materna de las personas sordas (Instituto Nacional para Sordos, INSOR, 2016) no se trata solo del ejercicio de traducción-retrotraducción (Sutton et al., 2019) -implica pensar en la poca riqueza léxica de la lengua de señas colombiana (LSC) - comparada con el español, los procesos cognitivos en personas sordas, la inequiparabilidad de conceptos, palabras y expresiones idiomáticas (Andrews y Mason, 1991).

La LSC tiene características diferentes a las lenguas orales, entendiendo que lengua y pensamiento estáníntimamente relacionados, las personas sordas señantes, aun estando dentro del mismo espacio geográfico, poseen procesos de pensamiento, construcciones cognitivas, cultura e identidad diferentes a las de habla castellana, por ello Melgar (2009) ahonda en diferencias que se deben considerar al hablar de psicología en personas sordas. Esta población representará en el 2020 un 1.1\% de los colombianos (INSOR, 2019) y existen familias que no disponen de información suficiente para acceder a servicios de salud, no se sienten incluidos, ni conocen sus derechos en salud (INSOR, 2018).

En consonancia al desarrollo del pensamiento, Decker et al. (2012) indican que una comunicación y lenguaje efectivos, favorece el desarrollo cognitivo y social en los niños como eje fundamental en la interacción social, su disfuncionalidad afecta algunos factores, como sus funciones cognitivas y limita la interacción de la persona con el mundo (Monsalve y Núñez, 2006). El lenguaje se vale de dos enfoques esenciales, la comunicación intersubjetiva e intrasubjetiva (Patiño, 2010); el primero hace referencia a la dimensión social y el segundo a la dimensión intrapersonal.

La edad en que inició la sordera (combinada con factores como el entorno familiar y social) es la variable que más condicionará el desarrollo cognitivo de la persona sorda, desde a estrecha relación entre lenguaje y pensamiento (Lane, 1984). Partiendo del impacto del lenguaje en el desarrollo cognitivo, una persona sorda, al carecer de competencia comunicacional, presenta vacíos en adquisición de información suministrada por su entorno inmediato, pues no todos los datos ingresan a su memoria. Tanto sus representaciones mentales, como expresión del pensamiento, dependen de la disponibilidad de dicha información en su lengua materna, el desarrollo del vocabulario en la persona sorda más la capacidad de los 
intérpretes que interactúan con la persona para transmitir un mensaje, contextualizar, explicar las situaciones y conceptos asociados al mensaje.

Por otra parte, en el contexto psicoterapéutico al momento de plantear el autorregistro de pensamientos propios del modelo Beckiano, evaluando emociones, pensamientos y conductas a partir de situaciones cotidianas y escribir cómo fueron experimentadas de forma particular por la persona (Beck, 1995, ver tabla 1), se encuentra la imposibilidad de generar escritura en LSC, en tanto es ágrafa, generando la necesidad de realizar ajustes en consonancia con las posibilidades y características de la LSC.

Tabla 1. Registro De Pensamientos automáticos

\begin{tabular}{|c|c|c|c|c|c|}
\hline $\begin{array}{l}\text { Día- } \\
\text { Hora }\end{array}$ & Situación & $\begin{array}{l}\text { Pensamiento } \\
\text { Automático }\end{array}$ & Emoción & $\begin{array}{l}\text { Respuesta } \\
\text { Adaptativa }\end{array}$ & Resultado \\
\hline & $\begin{array}{l}\text { ¿Qué } \\
\text { acontecimientos } \\
\text { reales lo } \\
\text { llevaron a esa } \\
\text { emoción? } \\
\text { ¿Qué } \\
\text { sensaciones } \\
\text { físicas } \\
\text { experimentó? }\end{array}$ & $\begin{array}{l}\text { ¿Qué } \\
\text { pensamientos } \\
\text { y/o imágenes } \\
\text { pasaron por su } \\
\text { mente? } \\
\text { ¿En qué } \\
\text { medida creyó } \\
\text { en cada uno } \\
\text { de ellos en ese } \\
\text { momento? } \\
\text { (Valoración } \\
0-100 \%)\end{array}$ & $\begin{array}{l}\text { ¿Qué emoción } \\
\text { sintió en ese } \\
\text { momento? } \\
\text { ¿Qué grado de } \\
\text { intensidad? } \\
\text { (Valoración } \\
0-100 \%)\end{array}$ & $\begin{array}{l}\text { ¿Qué distorsión } \\
\text { cognitiva } \\
\text { produjo? } \\
\text { ¿Cuánto cree } \\
\text { usted en cada } \\
\text { respuesta? } \\
\text { (Valoración } \\
0-100 \% \text { ) }\end{array}$ & $\begin{array}{l}\text { ¿Cuánto cree usted } \\
\text { ahora en cada PA? } \\
\text { (Valoración 0-100 } \\
\% \text { ) } \\
\text { ¿Qué emociones } \\
\text { siente } \\
\text { actualmente? } \\
\text { ¿Con qué grado de } \\
\text { intensidad? } \\
\text { (Valoración 0-100 } \\
\text { \%) } \\
\text { ¿En qué intensidad } \\
\text { quedó la anterior } \\
\text { emoción? } \\
\text { ¿Qué hará? }\end{array}$ \\
\hline
\end{tabular}

Nota: Registro de pensamientos disfuncionales. Copyright 1995 by Judith S, Beck. Tomado de Beck, J. (1995). Terapia cognitiva: conceptos básicos y profundización. Barcelona: Editorial Gedisa S.A.

Sobre ello, se ha planteado siguiendo a Mayer y Akamatsu (2003); Stewart, (1993) que la lengua de señas es ágrafa, mientras que la lengua oral tiene una forma escrita, en la que predomina la vía auditiva/oral y de poco acceso sintáctico a personas sordas. Así mismo, Gutiérrez y Salvador (2010) precisan sobre las dificultades en las lenguas de señas para establecer concordancia entre elementos de las frases, utilizar verbos adecuadamente, preposiciones, la estructura del complemento verbal y nexos conjuntivos en oraciones compuestas. Además, en ocasiones se pretende enseñar a los niños sordos la lectura y escritura de una lengua oral que les es conocida en forma fragmentaria y cuentan con menos conocimientos del mundo, por su adquisición del lenguaje (Alegría, 2010; Trezek et al., 2003).

Con relación a ello, explicar el modelo Beckiano para población sorda, im- 
plica pensar en sus procesos cognitivos y de aprendizaje, en que cada uno puede contar con capacidad comunicacional diferente, pero a su vez atraviesa situaciones que afectan su salud mental y que por tanto, son factores que pueden ser signo de intervención terapéutica. No obstante, el hecho de brindar cualquier psicoterapia sin ajustes razonables (Corte Constitucional T - 933 de 2013) ignoraría la Convención sobre los Derechos de las Personas con Discapacidad de la Organización de las Naciones Unidas; donde aseguran el ejercicio efectivo de los derechos de las personas con discapacidad, mediante la adopción de medidas inclusivas, acción afirmativa y de ajustes razonables que permitan a la población con discapacidad, disfrutar de servicios a los que acceden las personas sin discapacidad (ONU, 2008).

Se ha ido desarrollando el interés por pensar en prácticas psicoterapéuticas que consideren el contexto de pacientes con algún tipo de discapacidad. Tal es el caso de García (2004) quien se interesó por resaltar el lugar de la psicología especial en la atención a personas con necesidades educativas especiales, y que es una rama de la psicología que aún requiere de desarrollo y de atención desde la intervención clínica.

Es en este sentido que este ejercicio investigativo cobró relevancia, planteándose como objetivo principal, explicar el proceso de ajuste razonable del modelo de terapia cognitiva de Aaron Temkin Beck a una muestra no probabilística de 16 personas sordas, con diferentes niveles comunicacionales. El propósito de sistematizar esta experiencia no solo permite organizar la información sino identificar los aprendizajes críticos emergentes de los elementos objetivos y subjetivos que estuvieron en interacción en el proceso de ajuste.

Si bien no se encontraron antecedentes asociados a adaptación de modelos psicológicos para personas sordas, si se encontraron estudios enfocados en necesidades de traducir temas de utilidad para población sorda, como el caso de la traducción de leyes en el trabajo de Rodríguez (2017), con la propuesta de traducción del código de infancia y adolescencia. Otros estudios se han dirigido a explicar e ilustrar los procesos de traducción. Tal es el caso de Peluso (2015) con un proceso de traducción de la lengua Uruguaya a texto diferido, empleando videograbaciones, siguiendo una metodología de grabación por fragmentos-párrafos, que posteriormente se unen para darle mayor orden secuencial al mensaje traducido. Siguiendo otros métodos de traducción afirman que existen tres roles: el primero es el traductor, el segundo es el revisor y finalmente el corrector; que abordan elementos interlingüísticos e interculturales llevando a cabo once pasos preparación: reunión de proyecto, traducción inicial, revisión de la traducción, verificación y finalización, grabación del video borrador, revisión externa, grabación del video apuntador, marcación de las separaciones por fragmento, párrafo, grabación final y archivo de la traducción. Este trabajo al seguir fases de traducción y adoptar el uso de videograbaciones orienta metodológicamente el presente estudio.

Uno de los primeros trabajos documentados (Lindsay et al., 1997), aplicó un tratamiento a 2 personas con discapacidad intelectual mediante técnicas adaptadas 
del modelo de Beck, evidenciando cambios positivos en el Inventario de Ansiedad. McCabe et al.(2006) desarrollaron un programa de 5 semanas también a participantes con discapacidad intelectual cuyo propósito fue monitorear sus estados anímicos y reforzar comportamientos adaptativos mediante la reestructuración de distorsiones cognitivas. Los resultados demostraron la efectividad del programa en el tratamiento de la depresión en personas con (DI). McCabe et al. (2006) desarrollaron un programa de intervención desde el modelo de Beck durante 12 semanas dirigido a personas con (DI), se les enseñó a desarrollar y mejorar sus habilidades sociales y a reconocer sus pensamientos, emociones y comportamientos mediante ejemplos concretos, repetición y juegos de rol. Estos estudios indicaron que haciendo ciertas adaptaciones al modelo, se puede lograr un acercamiento efectivo al tratamiento.

En la revisión del libro "Terapia cognitiva-conductual para personas sordas y con problemas de audición” de Small (2009) se describen una serie de estrategias para adaptar este modelo de terapia en personas con déficits cognitivos y en el lenguaje, indicando el uso de metáforas, historias y juegos en el desarrollo de la terapia. Asimismo, se identifica la construcción de tarjetas visuales para ilustrar conceptos a personas sordas.

Son múltiples los modelos cognitivos, este trabajo se focalizó en el modelo de Beck, quien desarrolla La Terapia Cognitiva. Basada en la teoría de que la conducta y los afectos de una persona se determinan por su forma de estructurar el mundo (Beck, 1976).

El enfoque cognitivo se desarrolla sobre la concepción de diferentes modelos del procesamiento de la información para la cognición, uno de ellos es el modelo cognitivo de Beck et al. (1996) identificando que los individuos no responden inmediatamente a una situación sino que antes perciben, interpretan, evalúan, y asignan significado a lo que les sucede, con base en pensamientos, ideas y creencias previas. A esto se le conoce como la explicación del modelo cognitivo de Beck, entendiendo modelo como una concepción teórica para mirar el objeto que se estudia (García, 2015).

Las personas construyen diálogos internos, frases cortas que se dicen a sí mismos. Esto es denominado por Beck (1963) como pensamientos automáticos, los cuales pueden encasillarse en distorsiones cognitivas, estas son interpretaciones erróneas o sesgadas que los individuos realizan sobre los acontecimientos. Algunas de las distorsiones cognitivas son: razonamiento emocional, abstracción selectiva, catastrofización entre otros . Para explicar a las personas sordas cómo construyen sus diálogos internos se hace necesario adaptar la explicación del modelo y abordar los conceptos de discapacidad auditiva, personas sordas, modelos de enseñanza para personas sordas y lengua de señas.

Por discapacidad auditiva, se entiende cualquier pérdida de audición que implique intercambios comunicativos. La discapacidad auditiva se puede clasificar, según el momento en que se produzca la disminución, grado de pérdida, etiología, lugar de lesión y edad en que haya producido sordera (Consejo Nacional de Fomento 
Educativo, 2010). Así pues, el término sordo hace referencia a cualquier persona que presente dificultades para oír, su comunicación puede ser oral, dado puede tener un resto auditivo que le permite incorporar la lengua o ser una persona ensordecida que habría adquirido una lengua previa a la sordera, o en lengua de señas.

En 1993, la Federación Nacional de Sordos de Colombia (FENASCOL) publicó un manual de enseñanza, en donde se incluyen algunos aspectos lingüísticos de la LSC. A partir de 1996, año del reconocimiento legal de la lengua de señas en Colombia (Ley 324 de 1996), se suceden varios estudios en relación con algunos aspectos gramaticales de la lengua de señas. Es Oviedo (2001) quien en su libro Apuntes para una gramática de la Lengua de Señas Colombiana, hace el que hasta ahora es el más completo tratado de gramática sobre dicha lengua. En este se evidencia que la LSC puede ser estudiada como cualquier otra lengua y que posee características particulares, como se ilustra en el siguiente cuadro (tabla 2), donde se describe una clasificación de los diferentes tipos de señas.

Tabla 2. Tipos de señas

\begin{tabular}{|c|c|}
\hline \multicolumn{2}{|c|}{ Clasificación de la LSC } \\
\hline $\begin{array}{l}\text { Según las partes del cuerpo } \\
\text { involucradas (Oviedo, 2001) }\end{array}$ & $\begin{array}{l}\text { Según la entiedad representada (INSOR, } \\
2006 \mathrm{a} / \mathrm{b})\end{array}$ \\
\hline $\begin{array}{l}\text { Manuales: Se realizan con las manos } \\
\text { y generalmente una es pasiva y la otra } \\
\text { activa }\end{array}$ & $\begin{array}{l}\text { Pantomímicas: Representan exactamente la } \\
\text { acción que se describe }\end{array}$ \\
\hline $\begin{array}{l}\text { No manuales: Utilizan la expresión facila } \\
\text { en lugar de las manos para realizarse }\end{array}$ & $\begin{array}{l}\text { Imitativas: Seleccionan alguna parte del } \\
\text { significado completo para imitarlo o menos } \\
\text { cercanamente }\end{array}$ \\
\hline
\end{tabular}

A la hora de adaptar un instrumento como el modelo de terapia cognitiva de Beck a población sorda es importante pensar en todas estas particularidades de la lengua de señas y de sus usuarios para garantizar los Ajustes Razonables de los que habla la Convención de los Derechos de las Personas con Discapacidad (ONU, 2008), entendiendo ajuste razonable como las adecuaciones necesarias que no impongan una carga indebida para garantizar el ejercicio de sus derechos a las personas con discapacidad (Salazar, 2016).

\section{Método}

En bases de datos especializadas empleando el uso de tesauros: sordera, lengua de señas, psicoterapia, cognición, discapacidad, modelo de intervención. Asimismo, pero no se encontraron estudios de ajustes razonables de modelos teóricos o psicoterapéuticos en población sorda. Se seleccionaron trabajos con afinidad temática y cercanía a población sorda sin encontrar resultados aun así se estudió y siguió el protocolo de traducción, re-traducción e interpretación

Posteriormente se llevó a cabo la implementación de una metodología cualitativa, denominada sistematización de experiencias por medio de bitácoras donde 
se consignó el proceso de ajuste razonable del modelo Cognitivo de Aaron T. Beck a personas sordas usuarias de la LSC. El grupo de investigadores consignaba la síntesis de cada experiencia en un formato (ver tabla 3) y con los apuntes de equipo, se ordenaron las vivencias, se sintetizó lo vivido en fases denominadas “momentos", luego se realizó una interpretación crítica de este proceso y finalmente se extrajeron los aprendizajes obtenidos en dicho proceso.

\section{Participantes}

Los participantes fueron seleccionados de manera aleatoria , posterior a difundir la pretensión del estudio con la comunidad sorda se recopilaron participantes voluntarios, contando con una muestra no probabilística de 16 personas prelocutivas con sordera profunda, e hipoacusia tanto bilateral como lateral comprobado bajo orden médica, todos en edades entre 25 y 57 años de edad, mujeres y hombres, todos con localidad en el área metropolitana de Medellín- Colombia, estableciendo como criterios de selección que la población no tuviese otra discapacidad distinta a la sordera, ser mayor de 18 años y con una capacidad comunicacional suficiente en LSC, con diferente habilidad comunicacional , dos de los participantes eran postlingüísticas oralizados no usuarias de la L.S.C

\section{Instrumento}

Para la ejecución el método cualitativo denominado sistematización de experiencias se acudió a un proceso de toma de apuntes cronológicos conocido como Bitácora. Este instrumento se usó desde el inicio del estudio facilitando la sistematización de experiencia del proceso de ajuste para la explicación del modelo Beckiano a personas sordas señantes, por lo que el grupo de investigadores no solo valoraba la experiencia como fuente de aprendizaje sino que abstrajo, analizó y consignó los aspectos centrales o información relevante. Al finalizar la investigación se reconstruyó lo vivido ordenando y clasificando la información, para hacer una interpretación crítica del proceso. A continuación se presenta el formato de bitácora 1 y 2, donde se consignaron los acontecimientos vividos por el equipo investigador, durante el proceso de ejecución del proyecto. La bitácora, es identificada como el instrumento más utilizado en el marco de la metodología de sistematización de experiencias. 
Tabla 3. Formatos para el registro de información

\section{Formato 1: Bitácora}

Contexto de la situación: Contexto en que se dio el momento significativo, quiénes estaban presentes y en qué momento.

Relato de lo que ocurrió: (describiendo lo que sucedió, de forma que de cuenta del desarrollo de la situación o momento vivido por el equipo de investigadores)

\section{Formato 2: Bitácora}

Actividad Objetivo Pasos Dificultades Aprendizajes Sugerencias

\section{Procedimiento}

El resultado del diseño cualitativo denominado sistematización de experiencias a través de bitácoras pretenden ilustrar los aprendizajes organizados cronológicamente generados a lo largo del proceso.

Así pues, en consecuencia con el producto esperable de una sistematización, se describen aquí en cinco momentos experienciales tenidos en cuenta bajo criterios de selección del grupo de investigadores donde se ilustró cómo se efectuó paso a paso el ajuste razonable del modelo de terapia cognitiva propuesto por Aaron Beck para personas sordas señantes.

Una vez seleccionado el grupo de participantes, el primero momento experiencial fue analizar la pertinencia y necesidad de que el modelo cognitivo fuese ajustado a los participantes del estudio, sumado a esto se identificó la aplicabilidad del modelo Beckiano, acudiendo a un grupo de discusión conformado por la misma comunidad, personas sordas, modelos lingüísticos e intérpretes.

Una vez encontrada la aplicabilidad del modelo a esta comunidad, se inició con el segundo momento llamado de traducción-retrotraducción, esto para obtener una versión consensuada y fiel del texto original en la LSC. Este procedimiento ha sido empleado por (Lauffer et al., 2013) coincidiendo con nuestro estudio en que el principal enfoque de evaluación a la hora de adaptar un modelo como estos en otra lengua y cultura es la equivalencia semántica y cultural, no sin antes pasar por una etapa de definición teórica, para producir una versión conceptualmente equivalente del texto de origen en lengua Castellana.

En esta etapa de definición conceptual emergió la necesidad de realizar una capacitación en el Modelo Cognitivo de Beck a los intérpretes partícipes de este segundo momento, generando un encuadre de los alcances y limitaciones de su rol, de forma tal que no se modificara la intencionalidad del autor. Esta actividad se hizo con un grupo de intérpretes con experiencia en trabajo con la comunidad, considerando las limitaciones de la LSC, conservando su gramática, procuraron la traducción fiel del contenido teórico explicado por Beck; con la intención de dirigir la traducción a población sorda de nivel comunicacional amplio, y evitando la traducción literal, donde se mantiene el orden de cada palabra en el texto fuente, y se tienen a traducir según sus equivalentes más cercanos, sino comunicativa en donde se intenta que la forma y el sentido sean comprensibles para los destinata- 
rios (Newmark, 1982). Se reconocieron primero las señas disponibles, la intención del autor y cuidando que cada interpretación respetara las necesidades culturales, lingüísticas y cognitivas de la población a la que iba dirigida.

De este grupo se obtuvo la primera versión de la interpretación en formato de video en LSC, la cual fue llevada a un segundo grupo de intérpretes para que realizaran la voz de los videos sin estar capacitados en el modelo cognitivo, ni contextualizados en el tema. Se completó así un proceso de traducción y retrotraducción (Mapi Institute, 2019) entre ambos grupos de intérpretes, concebida por el grupo de investigadores como una técnica de verificación de la fidelidad de una traducción. Las retrotraducciones son técnicas comunes en las ciencias y la medicinas, en los casos de instrumentos, consentimientos informados y protocolos, en el cual se evalúa el grado de semejanza de la retraducción con la versión original, pues si no hay cambios significativos en la esencia e intencionalidad, se puede concluir que la traducción es adecuada (Sutton et al., 2019).

El proceso fue repetido hasta obtenerse la interpretación más fiel del modelo cognitivo explicada en formato de videos. Cada una de las modificaciones implicó borrar el material ya grabado y filmar videos nuevos, hasta llegar al consenso del mejor estructurado para la población.

El tercer momento experiencial consistió en socializar con personas sordas, post lingüísticas, con diferentes capacidades comunicacionales y modelos lingüísticos, obteniendo sugerencias al contenido del modelo explicativo de Beck en los formatos de video. Esto se asemeja al proceso de textualidad diferida efectuado en Uruguay sobre la traducción entre español escrito y la lengua de señas de dicho país, por medio de videograbaciones en las cuales es posible establecer una cultura letrada a través del uso recurrente de una textualidad diferida (Peluso, 2015).

El cuarto momento contó con la participación de una persona sorda técnica en edición de videos, quien diseñó del material, utilizando gráficos, dibujos e iconicidad para aumentar la comprensión del contenido en la población, teniendo en cuenta señas básicas conocidas e incorporando la actuación de modelos de lengua y cultura, los cuales son adultos sordos que realizan acompañamiento en el aula a los estudiantes de primaria y fuera de ella, donde socializan la LSC y elementos de la cultura sorda, dadas sus habilidades en LSC y en español escrito. Los videos fueron evaluados por 3 jurados quienes identificaban por medio del formato de evaluación, la pertinencia en la interpretación, claridad, suficiencia y semejanza al texto original. Sus sugerencias fueron acogidas y los videos nuevamente fueron grabados para pasar a la explicación del modelo ante los participantes.

En un quinto momento se identificó la comprensión del modelo cognitivo pidiendo la explicación de este por los participantes, logrando una interpretación del procesamiento de la información y distorsiones cognitivas de manera similar a la explicada por Aaron Beck. 


\section{Resultados}

Al finalizar la experiencia se obtuvieron cinco videos: descripción de emociones, activación corporal, procesamiento de la información, distorsiones cognitivas y control del estado de ánimo, cada uno de ellos con contenido gráfico y en LSC explicando el modelo cognitivo propuesto por Aaron T Beck, ilustrados de la siguiente manera:

Figura 1. Capturas de pantalla extraídas del video explicativo de emociones en LSC

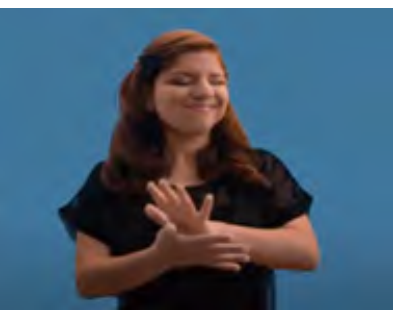

a

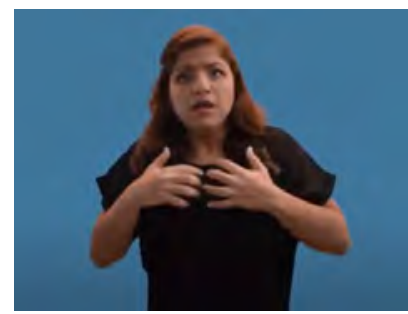

b

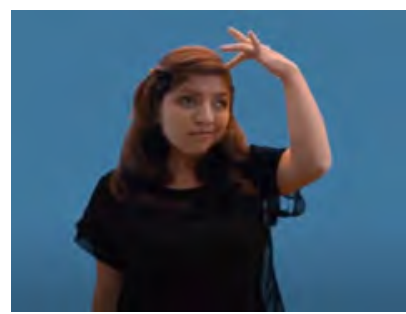

C

De izquierda a derecha en la figura 1: a) tranquilidad, b) angustia, y c) corresponde a una seña sin equivalente en español, que refiere a una emoción que puede interpretarse como "quedarse sin palabras o el deseo de escapar de un momento incómodo”.

Una vez ilustradas las emociones se explicaron cada una de las respuestas fisiológicas. A continuación (figura 2), se presentan algunas imágenes tomadas del video explicativo enseñado a los participantes.

Figura 2. Capturas de pantalla extraídas del video explicativo de emociones en LSC respuestas fisiológicas

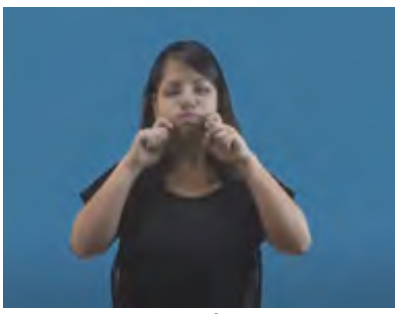

d

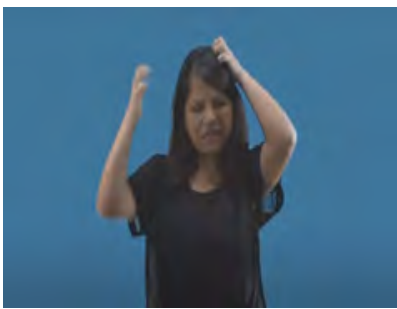

e

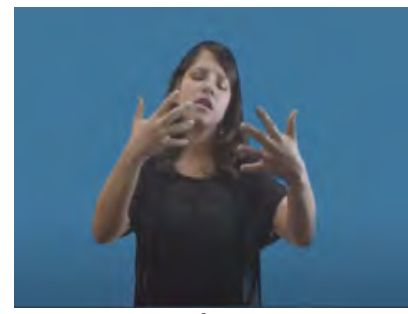

f

De izquierda a derecha: d) enrojecerse, e) rascarse la cabeza y f) mareo.

Ilustrada la información previa, se procede a explicar el procesamiento de la información en un tercer vídeo representado en la siguiente imagen (figura 3): 
Figura 3. Procesamiento de la información

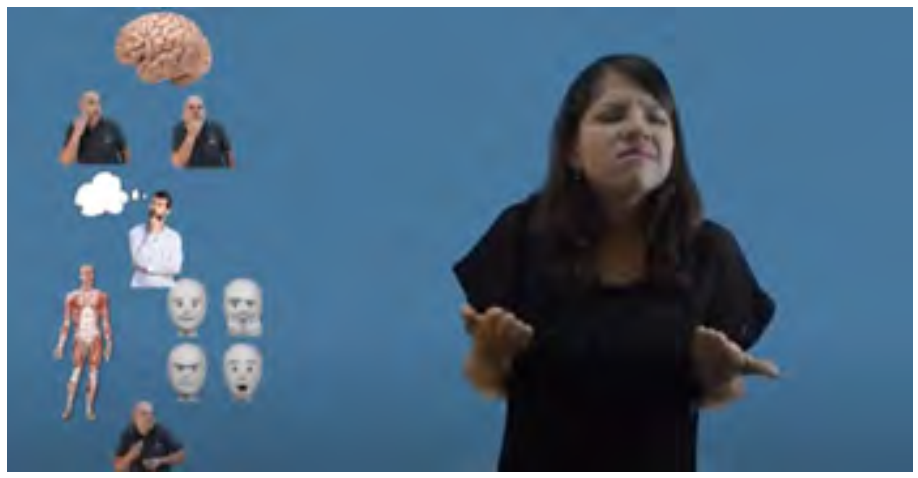

Para indicar el control del estado de ánimo se realizó una pirámide invertida (figuras 4,5 y 6), en su parte inferior se muestra el porcentaje $0 \%$ y en su parte más alta un $100 \%$ indicando a la persona sorda que señale el punto exacto en el que se encuentra el grado de la emoción experimentada.

Figura 4. Estado de ánimo en su estado más alto

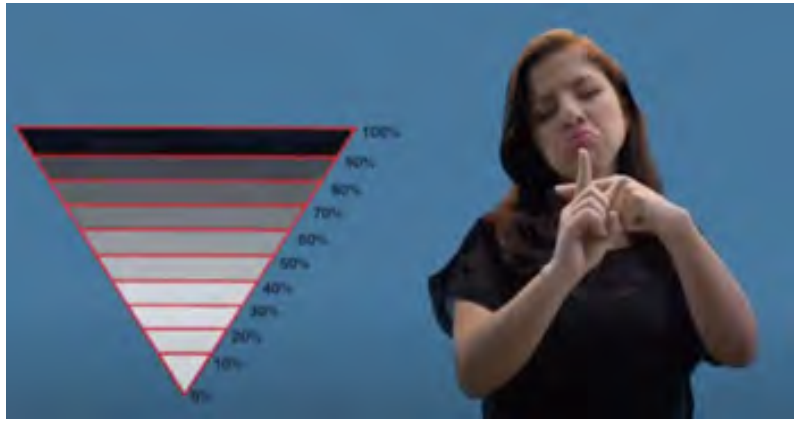

Figura 5. Estado de ánimo en un estado medio

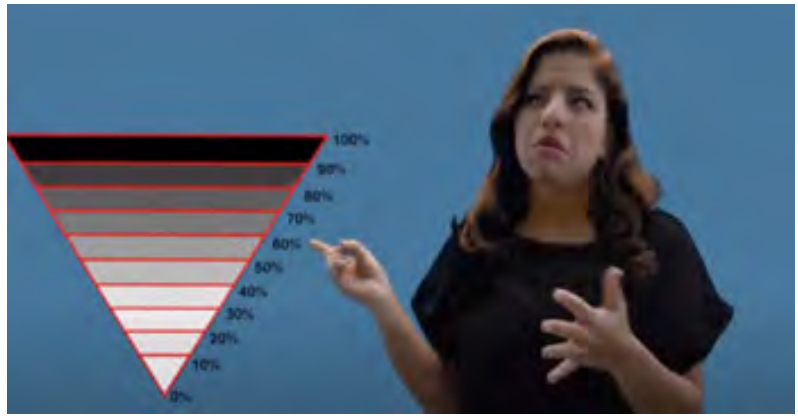


Figura 6. Estado de ánimo en el estado más bajo

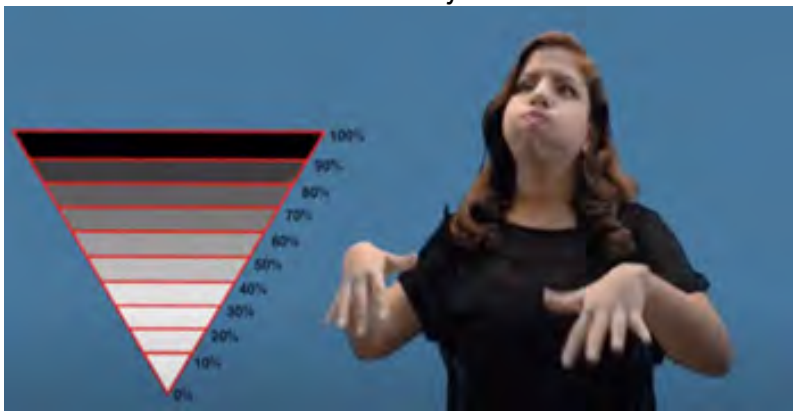

A continuación, se muestran capturas de pantalla de un cuarto video que ilustra una de las 15 distorsiones cognitivas que fueron adaptadas (figura 7). La imagen ilustra la explicación de la distorsión titulada magnificación, minimización en la cual, de una misma situación se escogen los rasgos negativos y se exageran, mientras que los aspectos positivos se minimizan.

Figura 7. Capturas de pantalla de distorsión magnificación

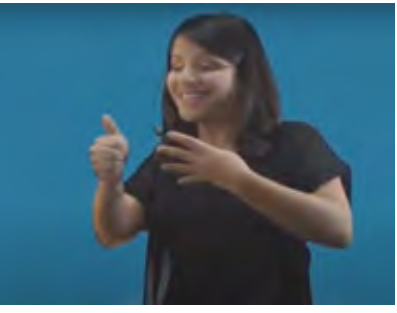

g

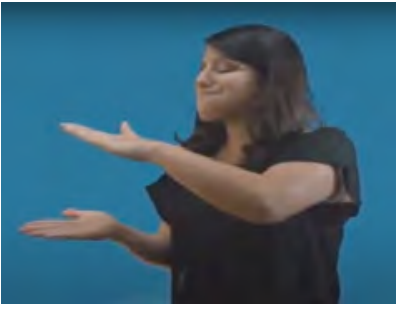

$\mathrm{h}$

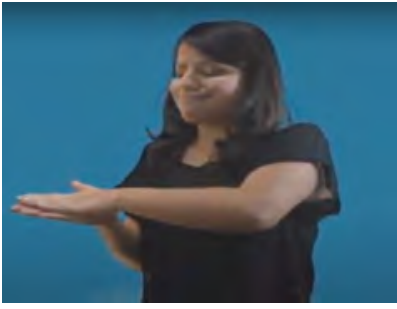

i

De izquierda a derecha: g) se muestran aspectos de una situación, h) Uno de los rasgos negativos se maximiza, e i) seña de quitar importancia, hasta quedar reducido a nada.

\section{Aprendizajes Significativos}

En el marco de diseño investigativo denominado sistematización de experiencia, los aprendizajes significativos de los investigadores son un resultado protagónico para dar cumplimiento a la interpretación crítica esperable para este tipo de investigación de enfoque cualitativo:

Al ilustrar el modelo cognitivo de Beck a los participantes seleccionados mediante su lengua materna, ellos brindan retroalimentación de lo comprendido, por tanto, ajustar el modelo cognitivo de Beck a una muestra de personas sordas usuarias de lengua de señas colombiana, permite que comprendan el contenido que se pretende transmitir.

En el video explicativo del modelo de procesamiento de la información, se hace necesario realizar ciertas adaptaciones, dadas las diferencias conceptuales 
entre el español y la Lengua de Señas Colombiana que deben ser tenidas en cuenta. Este aprendizaje se logró al reemplazar la seña "situación” por "qué pasó” pues el concepto de situación se asocia a problemas sociales o dificultades personales graves y no a eventos cotidianos, mientras que la seña "qué pasó” describe algo que sucede y de allí se deriva un pensamiento automático. Se encontró una tendencia en la muestra piloto a contar el problema desde su inició cuando se les pedía relatar solo una de las situaciones que componían el problema por lo que se podría analizar en próximos estudios cómo esto se relaciona con la teoría de la mente o sus procesos de asimilación de información teniendo en cuenta el desarrollo histórico de la lengua de señas en Colombia, su vocabulario construido hasta el momento y la comprensión del pensamiento y el lenguaje como proceso en paralelo.

Al presentar los tipos de emociones en formato de video, algunas señas no tienen un equivalente lingüístico en español, por ejemplo, las señas de: "explosivo”, “sentirse mal”, “dolor emocional” y sentirse "sin palabras”. Lo que amplió el espectro de comunicación sobre los sentimientos que las personas sordas comunicaban, cuando en español existen palabras que en lengua de señas no existe aún, se realizó un acuerdo con las personas sordas para diseñar la seña teniendo en cuenta la ergonomía de las manos y el significado. Pero cuando una seña existente no tenía equivalente al español facilita el trabajo en este tipo de intervenciones pues permite en este caso comprender exactamente qué emoción propia de la cultura sorda se está dando a comunicar.

Con las distorsiones cognitivas visión en forma de túnel y pensamiento dicotómico se encuentran dificultades de comprensión por parte de las personas sordas, es desde las expresiones faciales sarcásticas donde se encontró la manera de ejemplificar algunas de dichas distorsiones. El sarcasmo se ejemplifica a través de rasgos no manuales, particularmente la expresión facial, que algunos autores han comparado con la función de la entonación, un recurso también usado en lenguas orales para expresar sarcasmo. Todos estos rasgos no manuales han sido estudiados cuando se usan para transmitir emociones (Reilly et al., 1992).

En relación a las distorsiones cognitivas se logra identificar que la muestra no logró comprender la idea de que estas cumplen con las características de carencia de lógica y veracidad sino que asociaban este concepto a la generación de pensamientos negativos, al contrario, los pensamientos racionales no eran asociados a lógicos, veraces y comprobables, sino a pensamiento positivo esto tiene una relación directa con la capacidad comunicacional de las personas sodas y pobreza lingüística de las personas perteneciente a la muestra piloto. Cabe señalar en este aspecto que a menor vocabulario de la persona sorda mayor pensamiento concreto, menor capacidad de análisis y menor adquisición de información. Es por ello por lo que el terapeuta que utilice el instrumento debe aclarar a la persona sorda que una distorsión es un pensamiento errado, mientras que un pensamiento adecuado es un pensamiento lógico haciendo uso de las señas de pensamiento equivocado y pensamiento lógico. 


\section{Discusión}

Al finalizar la reflexión crítica de la experiencia se logra dilucidar que la adaptación del modelo cognitivo de Beck en personas sordas exige un análisis profundo de las características comunicacionales de cada persona, con base en ello se deben realizar los ajustes razonables para respetar su autonomía lingüística. Uno de los casos entre la multiplicidad de particularidades comunicacionales, son las personas sordas postlingüísticas oralizadas no usuarias de la lengua de señas colombiana, que hicieron parte de la experiencia investigativa. La oralización hace referencia a la capacidad de hablar, producto de un proceso de rehabilitación del habla y es por eso que para ellos el ajuste fue explicar el modelo de modo oralizado, sin la necesidad de ser acompañado por la LSC; sabiendo que su primera lengua es el español, tienen facilidad de lectura labio facial y muchos carecen de competencia en la comprensión de la lengua de señas. Para que las personas sordas que han sido oralizadas y usuarias de LSC entiendan el modelo cognitivo y pueda darse lugar a una terapia bajo este modelo, se debería hacer uso del español signado, en el cual se hacen señas usando la estructura gramatical del español (Escobar, 2015), por lo cual es posible bajo esta modalidad hablar (oralizar) y hacer señas a la vez permitiendo así el uso de dos canales: la lectura labio facial, y la comprensión de la lengua de señas en simultáneo. Por ende, todo ajuste razonable implica previamente estudiar la particularidad de la persona sorda a la que va dirigido y su preferencia comunicacional.

El lenguaje es considerado por (Luria, 1984) como instrumento para elaborar conocimiento, pero también como regulador de los procesos psíquicos, entre ellos el desarrollo del pensamiento. Por lo cual el equipo de investigadores reflexiona sobre la posible incapacidad de comprensión del modelo cognitivo propuesto por Beck en personas sordas sin una lengua o con niveles muy bajos de esta pues al pensamiento se accede por medio del lenguaje según la teoría de Luria (1994) en su libro pensamiento y lenguaje. En dichos casos, se sugiere explorar la posibilidad de recurrir a otro tipo de modelos de intervención, por ejemplo, el modelo conductual, en el que manejan otro tipo de códigos comunicacionales, diferentes al verbal, a través de mecanismos como los refuerzos positivos al interior de un programa de control de contingencias.

Durante la experiencia, el grupo de investigadores observó una limitación al llevar a cabo el ajuste del modelo a un grupo de personas sordas: su se denotó tendencia a ser influenciables por las opiniones de los demás al momento de generar respuestas. El trabajo grupal puede tornarse práctico, no obstante, durante la implementación de la estrategia explicativa es sugerible el trabajo personalizado, aunado a ello esto permite considerar las características singulares de la persona y poner en práctica lo planteado en el artículo 24 de la Convención de los Derechos de las Personas con Discapacidad, en el que se señala la necesidad de realizar ajustes razonables considerando las necesidades individuales de la persona; de allí a que sea más distante la brecha entre la respuesta a necesidades individuales y trabajos 
estandarizados y automatizados para la implementación de ajustes en población en situación de discapacidad.

En el modelo de Beck se suele recurrir a los ejemplos en los que se muestra una situación y diferentes formas de procesar la información respecto a la misma. Para las personas sordas al igual que en las oyentes se hace necesario recurrir a estos ejemplos, pues la sola explicación del modelo no basta. Los ejemplos ilustrativos que desee usar el terapeuta para explicar el modelo cognitivo o algunas distorsiones, deberán ser situaciones vividas por la persona a quien se explica el modelo, dado que, al optar por ejemplos hipotéticos, algunos participantes crearon supuestos más allá de lo que sugería la situación. Por el contrario, cuando se indicaron sus ejemplos personales la comprensión de la secuencia situación-pensamiento-emoción-conducta fue más evidente para la muestra.

Se sugiere también que la terapia cognitiva para personas sordas con una capacidad comunicacional poco amplia en lengua de señas, no se explique sólo con los instrumentos ya ajustados razonablemente en formato video. Este deberá complementarse con una dramatización icónica, ilustrando así una de las situaciones puntuales de la persona, sus pensamientos, sentimientos, sensaciones físicas y respuestas conductuales. Esto será necesario hasta que la persona sorda adquiera la habilidad metacognitiva que supone aplicar el modelo de Beck a su vida. Por el contrario, para sujetos con capacidad comunicacional amplia bastará con los instrumentos en formato vídeo de la explicación del modelo y un psicólogo usuario de LSC que logre ejemplificar con situaciones particulares de la persona sorda.

El equipo de investigadores del estudio expuesto en el presente manuscrito encontró que las personas sordas no pueden tener un registro de pensamientos como lo hacen los oyentes, se logra inferir que esto se debe a que presentan serias dificultades para manejar el español. según ya ha afirmado (Sánchez, 2008), se tendría que entrenar a las personas sordas en los diferentes tipos de distorsiones (ver figura 1) para que puedan llegar a incorporarlas con el repaso del material grabado. Asimismo, las personas sordas tienen la capacidad de generar pensamientos alternativos. Durante la explicación del modelo, no comprenden la diferencia entre estos y las distorsiones, pero a medida que va avanzando el proceso esta diferencia se clarifica a través de la práctica.

Algunas personas sordas de la muestra esperaron que las oyentes le suministrarán la respuesta a las preguntas ¿qué fue lo primero que vino a tu mente? y ¿cuál es el tipo de distorsión cognitiva?, Además esperaban un modelo basado en la consejería por parte de la psicóloga que se encontraba presente -se le solicitaba indicar cual sería la conducta funcional en situaciones similares futuras-, a esto, los investigadores reaccionaron partiendo de la premisa de que el objetivo de la terapia cognitiva es generar proceso de conciencia para que la persona logre tener una mayor conciencia y control de sus pensamientos (Beck, 1995) con las conductas que de allí derivan y no esperar a que se les indique cómo deben comportarse.

$\mathrm{Al}$ reflexionar sobre las vicisitudes señaladas anteriormente se puede iden- 
tificar que aun así las personas sordas de cualquier edad están en la capacidad de entender el modelo cognitivo y aplicarlo a sus vidas. El factor determinante para que una persona sorda pueda acceder a esta terapia es el manejo de la lengua de señas considerando el registro y de las señas usadas por la persona sorda.

El modelo cognitivo logró ser comprendido por personas sordas de capacidad comunicacional media en adelante, sin embargo, el proceso de comprensión de la información puede tomar más sesiones comparativamente a los oyentes, bajo el requerimiento de ilustraciones y ejemplos.

\section{Conclusiones}

Es poco lo que se ha hablado en relación con las prácticas de transferencia entre lenguas de señas y lenguas orales desde la teoría de la traducción e interpretación. Para esta sistematización adoptamos el concepto de traducción y no de interpretación, apoyados en el hecho que el ejercicio comprendía analizar el texto escrito y formular varias versiones de este en LSC con la mayor precisión posible y en un plazo de tiempo determinado, a diferencia de la interpretación, que se hace en tiempo real y con plazos de entrega más inmediatos. Aún así, esto es algo que aún está por discutirse, pues del concepto de traducción de lenguas de señas ha sido propuesto solo para personas sordas, usuarias del español escrito y de las lenguas de señas. El ejercicio de traducir y retrotraducir, para comparar con el texto original es una práctica que permite comprobar la fidelidad de una traducción y que ha sido usado en adaptaciones interlingüísticas e interculturales de diversos instrumentos especialmente, en las ciencias de la salud y que bien puede ser usado en contextos en los que el margen de error sea mínimo y podría ser usado, sistematizado cuando se quiera prestar servicios a personas sordas usuarias de lenguas de señas, de forma que se garantice la accesibilidad a una información presentada a la medida de sus características lengua y cultura con un mensaje dotado de semejanza al original dentro de las posibilidades que genera la lengua de señas.

La sola adaptación o ajuste de un formato, documento o en este caso explicación del modelo no basta para que el mensaje sea recibido y comprendido pues el desarrollo del lenguaje y el pensamiento se encuentran estrechamente conectados, dicha relación fue también evidente con las personas sordas que participaron en el proceso de ajuste razonable. A menor competencia comunicacional mayor dificultad para comprender el contenido de los videos; un requerimiento de mayor explicaciones del intérprete y más dificultad para exteriorizar sus pensamientos.

Teniendo presente el nivel comunicacional de cada participante (sin capacidad comunicacional en lengua de señas colombiana, con capacidad comunicacional básica, con capacidad comunicacional suficiente y con capacidad comunicacional amplia), al indicarles la secuencia del procesamiento de la información propuesta por el modelo cognitivo que relaciona los pensamientos, emociones y conductas, se hace comprensible toda vez que se tengan en cuenta las características comunicacionales de los participantes, el grupo de investigadores del presente estudio 
logra reflexionar sobre la posible conexión entre el desarrollo del lenguaje y la comprensión de cualquier tema incluyendo la explicación del modelo cognitivo pues desde la perspectiva de Luria (1994) pensamiento y lenguaje son proceso en paralelo bajo esta teoría entonces a menor vocabulario y capacidad comunicacional, menor comprensión del modelo ilustrado en el contenido de los videos lo que no ocurre con persona sorda de capacidad amplia y suficiente en lengua de señas colombiana.

Lo anterior ocurre naturalmente bajo el sustento teórico de (Barrachina et al., 2013) el lenguaje requiere de ayuda en habilidades cognitivas como la atención pensamiento y memoria, desde el autor el lenguaje es un moderador cognitivo por ello los trastornos de lenguaje se asocian a limitaciones intelectuales. El lenguaje, además, expresa lo que las personas tienen en su mente, sus creencias, deseos y sus pensamientos fundamentados en todos los conocimientos incorporados a través de su interacción con el medio.

Se podría puntualizar como indicador de desarrollo del pensamiento que a mayor grado de internalización de la lengua de señas, mejor será su capacidad de resolución de tareas y la capacidad, tal vez, de generar medios de pensamiento más abstracto, mejores estrategias de planeación y de auto monitoreo de su conciencia sirviéndose de la lengua de señas con su particular gramática y vocabulario (Barrachina et al., 2013), por lo que personas sordas con estos elementos comprenden adecuadamente la explosión del modelo cognitivo planteado por Beck después de adecuar los ajustes razonables requeridos en un proceso juicioso de traducción, retraducción adaptación.

\section{Referencias}

American Psychological Association (2012). Entendiendo la psicoterapia. http://www.apa.org/centrodeapoyo/ entendiendo-la-psicoterapia

Andrews, J y Mason, J. (1991). Strategy usage among deaf and hearing readers [Uso de la estrategia entre lectores sordos y oyentes]. Exceptional children, 57(6), 536-545. https://doi.org/10.1177\%2F001440299105700607

Alegría, J. (2010). Los lenguajes del niño sordo: for your eyes only. En M. Carrillo Gallego, y A. Domínguez Gutiérrez (Coords.), Dislexia y sordera: líneas actuales en el estudio de la lengua escrita y sus dificultades (pp. 171-200). El Aljibe.

Barrachina, L., Aguado, G., Cardona i Pera, M. y Sanz-Torrent, M. (2013). El trastorno especifico del lenguaje, diagnóstico e intervención. Editorial UOC.

Beck, A. T. (1963). Thinking and depression: 1. Idiosyncratic content and cognitive distortions [Pensamiento y depression: 1. Contenido idiosincrático y distorsiones cognitivas]. Archives of General Psychiatry, 9(4), 324-333. https://doi.org/10.1001/archpsyc.1963.01720160014002

Beck, A. T. (1976). Cognitive therapy and emotional disorders [Terapia Cognitiva y Trastornos Emocionales]. International Universities.

Beck, A., Rush, J., Shaw, B. y Emery, G. (1979). Terapia cognitiva de la depresión. Desclée de Brouwer.

Beck, J. (1995). Terapia cognitiva: conceptos básicos y profundización. Gedisa.

Beck, A. T., Steer, R. A. y Brown, K. G. (1996). Manual for the Beck depression Inventory [Manual del inventario de depression de Beck] ( $2^{\mathrm{a}}$ ed.). The Psychological Corporation.

Bogiaizian, D. (1998). Tratamiento integrado de psicofarmacoterapia y terapia cognitivo-conductual en el trastorno de pánico con agorafobia. Revista de psicoterapia, 9(36), 43-54. https://tienda.revistadepsicoterapia.com/ tratamiento-integrado-de-psicofarmacoterapia-y-terapia-cognitiva-conductual-en-trastorno-de-panicocon-agorafobia.html 
Consejo Nacional de Fomento Educativo. (2010). Discapacidad auditiva guía didáctica para la inclusión en educación inicialy básica.https://www.gob.mx/cms/uploads/attachment/file/106806/discapacidad-auditiva.pdf

Corte Constitucional. (2013). Sentencia T-933/13. Gaceta de la Corte Constitucional [G.C.C.]. Colombia

Decker, K., Vallotton, C. y Johnson, H. (2012). Parents' communication decision for children with hearing loss: sources of information and influence [Decisión de comunicación parental para niños con pérdida auditiva: Recursos de información e influencia]. American annals of the deaf, 157(4), 326-339. https://doi. org/10.1353/aad.2012.1631

De la Fuente, O. (2014). Las personas sordas como minoría cultural y lingüística. Dilemata, 15, 267-287. https:// www.dilemata.net/revista/index.php/dilemata/article/view/308

Escobar, L. (2015). La lengua de señas mexicana ¿Una lengua en riesgo? Estudios de lingüística aplicada, 62, 125-152. https://doi.org/10.22201/enallt.01852647p.2015.62.420

García, M. E. (2004). La psicología en la atención a personas con discapacidad. Educ. rev, 23, 355-362. https:// doi.org/10.1590/0104-4060.346

García, F. (2015). Tres modelos teóricos generales en sociología: una des-unidad articulada. Revista española de investigaciones sociológicas, 151, 65-82.

Gutiérrez, R. y Salvador, F. (2010). Procesos cognitivos y metacognitivos en la expresión escrita de alumnos sordos de educación secundaria. En M. Carrillo Gallego y A. Domínguez (Eds.), Dislexia y sordera: líneas actuales en el estudio de la lengua escrita y sus dificultades (pp.101-110). El Aljibe.

Instituto Nacional para Sordos (2006a). Educación bilingüe para sordos, etapa escolar. Ministerio de Educación Nacional.

Instituto Nacional para Sordos (2006b). Diccionario básico de la Lengua de Señas Colombiana. Ministerio de Educación Nacional.

Instituto Nacional para Sordos (2018). De la garantía a la realización del derecho a la salud en las personas sordas: análisis de dificultades y oportunidades. Colombia. http:/www.insor.gov.co/bides/wp-content/ uploads/archivos/garantia_realizacion_der_salud_per_sordas.pdf

Instituto Nacional para Sordos (2019). Plan estratégico institucional 2019-2022. Colombia. http://www.insor. gov.co/home/descargar/plan_estrategico_INSOR_2019_2022V1.pdf

Lane, H. (1984). When the mind hears. A history of the deaf. Penguin Books.

Lauffer, A., Solé, L., Bernstein, S., Lopes, M. y Francisconi, C. (2013). Cómo minimizar errores al realizar la adaptación transcultural y la validación de los cuestionarios sobre calidad de vida: aspectos prácticos. Revista de gastroenterología de Mexico, 78(3), 159-176. https://doi.org/10.1016/j.rgmx.2013.01.008

Congreso de Colombia (2006). Ley 1090. Código deontológico y bioético y otras disposiciones. https://legislacion. vlex.com.co/vid/psicologia-dicta-deontologico-bioetico-60054118

Lindsay, W. R., Neilson, C. y Lawrenson, H. (1997). Cognitive-behaviour therapy for anxiety in people with learning disabilities [Terapia cognitiva-conductual para anxiedad en personas con discapacidad de aprendizaje]. En B. Stenfert Kroese, D. Dagnan y K. Loumidis (Eds.), Cognitive-behaviour therapy for people with learning disabilities (pp. 124-140). Routledge. https://doi.org/10.4324/9780203977200

Luria, A. (1984). Conciencia y lenguaje. Ediciones Universidad de Moscú.

Mapi Institute. (2019). Metodología. https://www.mapi-institute.com/linguistic-validation/methodology

Mayer, C. y Akamatsu, C. T. (2003). Bilingualism and literacy [Bilingüismo y alfabetización]. En M. Marschark y P. E. Spencer (Eds.), Oxford Handbook of Deaf Studies, Language and Education (pp. 136-147). Oxford University Press.

McCabe, M., McGillivray, J. y Newton, D. (2006). Effectiveness of treatment programmes for depression among adults with mild/moderate intellectual disabilities [Efectividad de los programas de tratamiento para depression en adultos con discapacidad intelectuales medias/moderadas]. Journal of Intellectual Disability Research, 50(4), 239-247. https://doi.org/10.1111/j.1365-2788.2005.00772.x

Melgar, J. (2009). Lenguaje, pensamiento y psicología del sordo. http://www.cultura-sorda.org/wp-content/ uploads/2015/03/Melgar_Lenguaje_Pensamiento_y_Psicologia_del_Sordo_2010.pdf

Ministerio de Salud. (1993). Resolución $N^{\circ}$ 008430. Por la cual se establecen las normas cientificas, técnicas y administrativas para la investigación en salud. Recuperado de: https://www.unisabana.edu.co/fileadmin/ Documentos/Investigacion/comite_de_etica/Res_8430_1993_-_Salud.pdf

Monsalve, A. y Núñez, F. (2006). La importancia del diagnóstico e intervención temprana para el desarrollo de los niños sordos. Los programas de detección precoz de la hipoacusia. Psychosocial Intervention, 15(1), 7-28

Newmark, P. (1982). Approaches to Translation [Enfoques de la traducción]. Pergamon Press

Oviedo, A. (2001). Apuntes para una gramática de la lengua de señas colombiana. INSOR -UNIVALLE.

Organización Mundial de la Salud. (2017). Salud y derechos humanos. https://www.who.int/es/news-room/factsheets/detail/human-rights-and-health 
Organización de Naciones Unidas. (2008). Convención sobre los derechos de las personas con discapacidad: Material de promoción. https://www.ohchr.org/Documents/Publications/AdvocacyTool_sp.pdf

Patiño Guiraldo, L. E. (2010). La lengua de señas colombiana como mediadora en el proceso de conceptualización de nociones relacionadas con las ciencias sociales en niños y niñas no oyentes [Tesis doctoral, Centro de estudios avanzados en niñez y juventud. Universidad de Mnizales-Cince]. https://repository.cinde.org.co/ bitstream/handle/20.500.11907/515/PatinoGiraldoLuzElena2010.pdf?sequence=1\&isAllowed=y

Trezek, B. J., Wang, Y. y Paul, P. V. (2003). Processes and Components of Reading [Procesos y componentes de la lectura]. En M. Marschark y P. E. Spencer, (Eds.), Oxford Handbook of Deaf Studies, Language and Education (pp. 99-114). Oxford University Press

Peluso, L. (2015). Traducción entre español escrito y lengua de señas uruguaya videograbada: un nuevo desafío. Cadernos de Tradução, Florianópolis, 35(2), 479-504.

Reilly, J., Mcintire, M. y Seago, H. (1992). Affective Prosody in American Sign Language [Prosodia afectiva en lenguaje de signos americano]. Sign Language studies, 75, 113 - 128.

Rodríguez, A. M. (2017). Propuesta inicial de traducción del código de infancia y adolescencia colombiano a lengua de señas. Revista Ratio Juris, 12(25), 23-42. https:/publicaciones.unaula.edu.co/index.php/ratiojuris/article/view/450/480

Salazar, E. R. B. (2016). La idea de los ajustes razonable como forma complementaria para conseguir la igualdad de las personas con discapacidad. Actualidad Jurídica-Universidad del Norte, 8, 40-54. https:// www.uninorte.edu.co/documents/4368250/0/La+idea+de+los+ajustes+razonables+como+forma+com plementaria+para+conseguir+la+igualdad+de+las+personas+con+discapacidad/4902d4f0-09bb-45b9af7c-ac0e341021ff?version=1.0

Sánchez, C. (2008). Que veinte años no es nada. La educación de los sordos en la encrucijada. Cultura Sorda. http://www.cultura-sorda.org/la-educacion-de-los-sordos-en-la-encrucijada/

Small, K. (2009). Therapy for deaf and hearing persons with language and learning challenges [Terapia para personas con sordas y oyentes con problemas de aprendizaje]. Cognitive behavioral therapy book reviews, 5(5). https://www.the-iacp.com/assets/CBTBR/cbtbr-vol_55d.pdf

Stewart, D. A. (1993). Bi-Bi to MCE? American Annals of the Deaf, 138(4), 331-337. https://doi.org/10.1353/ aad.2012.0384

Sutton, L., Ortiz, A. y Gatica, F. (2019). Evaluación De Las Competencias Docentes En Las Ciencias De La Salud (1 ${ }^{\mathrm{a}}$ ed.). El Manual Moderno. 